in biological fluids: do they represent the future of cancer diagnostics? Clin. Chem. 49:1272-1275.

14. Sorace, J.M., and Zhan, M. 2003. A data review and re-assessment of ovarian cancer serum proteomic profiling. BMC Bioinformatics. 4:24

15. Zhang, Z., et al. 2004. Three biomarkers identified from serum proteomic analysis for the detection of early stage ovarian cancer. Cancer Res. 64:5882-5890.
16. Lowenthal, M.S., et al. 2005. Analysis of albuminassociated peptides and proteins from ovarian cancer patients. Clin. Chem. 51:1933-1945.

17. Liotta, L.A., et al. 2005. Importance of communication between producers and consumers of publicly available experimental data. J. Natl. Cancer Inst. 97:310-314.

18. Villanueva, J., et al. 2006. Differential exoprotease activities confer tumor-specific serum peptidome patterns. J. Clin. Invest. 116:271-284. doi:10.1172/ JCI26022.

19. Zhou, M., et al. 2004. An investigation into the human serum "interactome". Electrophoresis. 25:1289-1298.

20. Lopez, M.F., et al. 2005. High-resolution serum proteomic profiling of Alzheimer disease samples reveals disease-specific, carrier-protein-bound mass signatures. Clin. Chem. 51:1946-1954.

\title{
Is the small heat shock protein $\alpha B$-crystallin an oncogene?
}

\author{
Sofia K. Gruvberger-Saal ${ }^{1}$ and Ramon Parsons ${ }^{2}$
}

1Department of Oncology, Lund University Hospital, Lund, Sweden. 'Departments of Pathology and Medicine, Institute for Cancer Genetics, Herbert Irving Comprehensive Cancer Center, Columbia University College of Physicians and Surgeons, New York, New York, USA.

In the last 5 years, global gene expression profiling has allowed for the subclassification of the heterogeneous disease of breast cancer into new subgroups with prognostic significance. However, for most subgroups, the nature of the contributions of individual genes to the clinical phenotypes remains largely unknown. In this issue of the JCI, Moyano and colleagues further examine the oncogenic potential of the small heat shock protein $\alpha$-basic-crystallin, commonly expressed in tumors of the basal-like breast cancer subtype associated with poor prognosis, and show that it is an oncogenic protein in the breast (see the related article beginning on page 261).

\section{Small heat shock proteins}

$\alpha$-Basic-crystallin ( $\alpha$ B-crystallin) is a member of the mammalian small heat shock protein (sHsp) superfamily, whose 10 -protein membership is defined by the presence of a conserved, approximately 90 -aa region (termed the $\alpha$-crystallin core) with divergent amino- and carboxyterminal domains (1-3). The sHsps function as cytoprotective molecular chaperones, preventing stress-induced aggregation of denaturing proteins as well as keeping aggregation-prone proteins in reservoirs of nonnative refoldable intermediates by holding proteins within large, soluble, multimeric structures. sHsps also appear to have a structural role; for example, $\alpha B$-crystallin together with $\alpha$-Acidic-crystallin comprise as much as $40 \%$ of the cytoplasmic proteins in lens cells of the eye and are thought to play an essential role in maintaining its

Nonstandard abbreviations used: $\alpha B$-crystallin, $\alpha$-basic-crystallin; CK, cytokeratin; ER, estrogen receptor; ErbB2, erythroblastic leukemia viral oncogene homolog 2; IHC, immunohistochemistry; MEK, MAPK kinase; sHsp, small heat shock protein.

Conflict of interest: The authors have declared that no conflict of interest exists.

Citation for this article: J. Clin. Invest. 116:30-32 (2006). doi:10.1172/JCI27462. transparency. However, in other tissues in the human body the $2 \alpha$-crystallins are expressed constitutively at much lower levels. $\alpha B$-Crystallin shares a close homology with another sHsp, Hsp27, and both of these proteins are activated in response to stresses such as heat shock, radiation, oxidative stress, and exposure to anticancer drugs. Moreover, both proteins have been shown to have antiapoptotic functions by interfering with the activity of various apoptotic proteins (2). Indeed, the literature suggests that $\alpha \mathrm{B}$-crystallin is important in the pathology of cancer, as overexpression of $\alpha \mathrm{B}$-crystallin has been observed in gliomas (4), renal carcinomas (5), breast carcinomas $(6,7)$, and ductal carcinoma in situ compared with matched normal breast tissue (8). Until now, the importance of $\alpha \mathrm{B}$-crystallin in cancer has been vaguely attributed only to its antiapoptotic functions.

\section{$\alpha B-C r y s t a l l i n$ as an oncoprotein}

In this issue of the JCI, Moyano and colleagues demonstrate properties of $\alpha \mathrm{B}$ crystallin not previously described to our knowledge that define it as a potential oncogenic protein (9). Using various cellculture systems, including reconstituted 3D basement membrane cultures, they report that constitutive overexpression of $\alpha \mathrm{B}$-crystallin in 2 human mammary epithelial cell lines, MCF-10A and MCF-12A, induced neoplastic-like changes such as EGF- and anchorage-independent growth, loss of polarity, disorganized acinar structure, increased proliferation, diminished apoptosis, and increased migration and invasion (Figure 1). These morphological changes were reliant on $\alpha \mathrm{B}$-crystallin overexpression, since retroviral RNA interference-mediated silencing of $\alpha B$-crystallin expression suppressed the abnormal phenotype. Moreover, although this transformation induced the expression and phosphorylation of ERK1/2, AKT, and p38, the authors show that the neoplastic changes were dependent on the ERK/MAPK pathway, as inhibition of only this pathway by highly specific MAPK kinase (MEK) inhibitors completely negated transformation. Transformation also appears to be dependent on the phosphorylation state of $\alpha B$ crystallin, as a pseudophosphorylation mutant of $\alpha \mathrm{B}$-crystallin, which mimics stress-induced phosphorylation, did not confer neoplastic changes. Furthermore, the authors demonstrate that $\alpha \mathrm{B}$-crystallin meets one of the "gold standards" for classification as an oncogenic protein: human mammary epithelial cells overexpressing WT $\alpha \mathrm{B}$-crystallin were shown to form invasive carcinomas in nude mice. In contrast, constitutive overexpression of the recognized oncogenes H-RasV12, cyclin D1, and erythroblastic leukemia viral oncogene homolog 2 (ErbB2) in these cells does not induce tumors in nude mice (10-12), suggesting that $\alpha \mathrm{B}$-crystallin is a more potent oncoprotein in this model. Finally, the authors demonstrate the clin- 


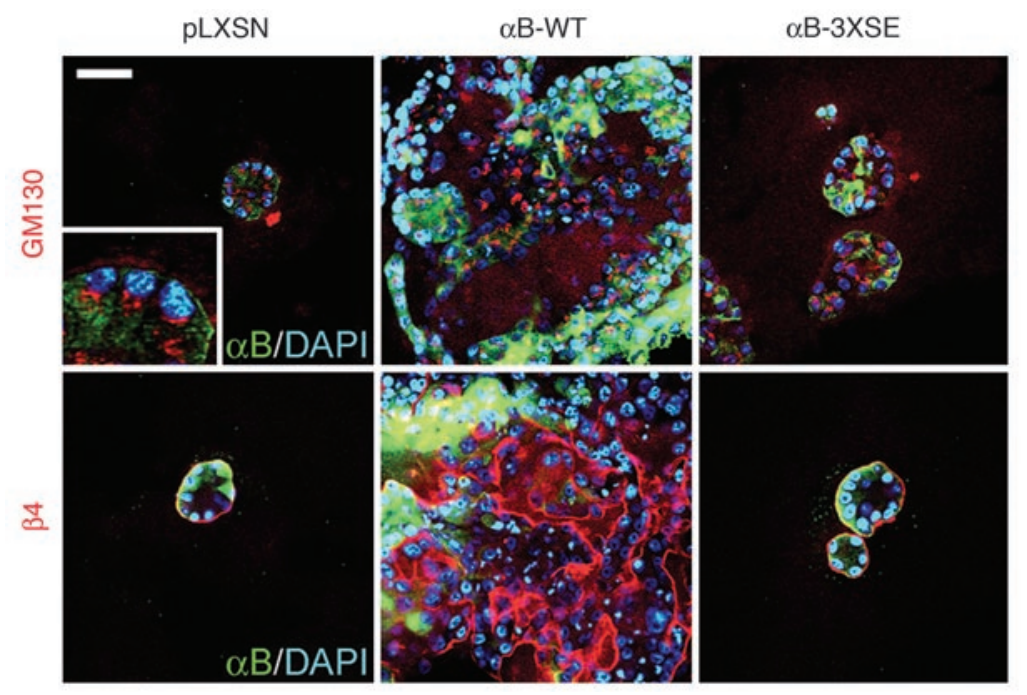

Figure 1

$\alpha \mathrm{B}$-Crystallin overexpression causes neoplastic-like transformation of human mammary epithelial cells. MCF-10A, immortalized human mammary epithelial cells, form acinar-like structures consisting of a single layer of polarized cells surrounding a hollow lumen, resembling normal breast ducts, when grown in 3D basement membrane culture. Activation of oncogenes such as ErbB2 induces neoplastic-like changes in these mammary acini. In this issue of the $\mathrm{JCl}$, Moyano et al. (9) demonstrate that MCF-10A cells stably overexpressing WT $\alpha B$-crystallin $(\alpha B-W T)$ form large, abnormal acini with filled lumen in contrast to the normal acini formed by empty vector-infected control cells ( $\mathrm{pLXSN}$ ) and cells overexpressing a pseudophosphorylation mutant of $\alpha B$-crystallin ( $\alpha B-3 X S E)$. Immunostained cross sections of the acini displayed higher levels of $\alpha \mathrm{B}$-crystallin (green) in $\alpha \mathrm{B}-\mathrm{WT}$ acini and marked disruption of normal acinar polarity, as evidenced by the localization pattern of the apical marker GM130 (red; upper panels) and the basolateral marker integrin $\beta 4$ (red; lower panels), compared with $\mathrm{pLXSN}$ and $\alpha \mathrm{B}-3 \mathrm{XSE}$ cells. In addition, the dramatic loss of polarity and disruption of acinar morphology in $\alpha B$-crystallin-overexpressing MCF-10A cells were also accompanied by increased EGF- and anchorageindependent growth, increased proliferation, diminished apoptosis, and increased migration and invasion, and these cells formed tumors in nude mice. These findings clearly indicate that $\alpha \mathrm{B}$-crystallin is an oncogenic protein in human mammary epithelial cells.

ical relevance of examining $\alpha B$-crystallin expression, as breast carcinomas positive for $\alpha \mathrm{B}$-crystallin (39 of 361 patients) were associated with significantly worse disease-specific survival independent of other commonly used prognostic markers. However, whether $\alpha \mathrm{B}$-crystallin truly meets the requirements of an oncogene is yet to be determined. Genetic studies demonstrating tumorigenic alterations of $\alpha \mathrm{B}$-crystallin such as mutations, amplifications, and translocations in human breast tumors and the generation of mammary tumors in transgenic $\alpha B$-crystallin-expressing mouse models would provide unequivocal evidence that $\alpha \mathrm{B}$-crystallin is a bona fide oncogene.

\section{Regulation of $\alpha$ B-crystallin and its targets}

Moyano et al. (9) bring the importance of $\alpha \mathrm{B}$-crystallin in breast cancer to the forefront, but it is still unknown whether $\alpha \mathrm{B}$-crystallin overexpression in breast tumors is driven by promoter transactivation, loss of transcriptional inhibition, increased DNA copy number, or other means, such as mutation of promoter elements. Moreover, it is not yet known whether overexpression of $\alpha \mathrm{B}$-crystallin is a major initiating oncogenic change in vivo. And what is the mechanism of the $\alpha B$-crystallin-dependent activation of the ERK/MAPK pathway? One may speculate that $\alpha \mathrm{B}$-crystallin stabilizes an activator of the pathway, such as Ras GTPases or one of the Rafs, or enhances ubiquitination and degradation of a MEK inhibitor, such as Raf kinase inhibitor protein, or have a completely novel function. The relationship between $\alpha \mathrm{B}$-crystallin and the ERK/MAPK pathway may also be cell type specific or context dependent, as a recent report (13) shows that in rabbit lens cells, in contrast to Moyano et al.'s observations in human mammary epi- thelial cells (9), $\alpha$ B-crystallin inhibits the ERK/MAPK pathway, thereby disrupting calcium-activated, p53-dependent apoptosis. In this respect, it is interesting to note that mutation of $\mathrm{p} 53$ occurs in over $80 \%$ of basal-like breast tumors, a rate much higher than most other subtypes (6). Furthermore, since ERK/MAPK has over 70 known substrates and modulates many fundamental cellular processes (e.g., cell proliferation, survival, differentiation, apoptosis, motility, and metabolism) (14), it remains to be seen which of these effectors are important in $\alpha \mathrm{B}$-crystallin-mediated transformation of mammary epithelial cells.

\section{$\alpha B-C r y s t a l l i n$ as a marker for basal-like tumors}

In the 1980s, it was first reported that breast tumors could be classified based on their expression of various epithelial cell cytokeratins (CKs), which are highly conserved cytoskeletal structural polypeptides found in all epithelial tissues. In these early studies, tumors that expressed the stratified CKs (such as CK5, CK14, and CK17) of the basal/myoepithelial cell lineage were associated with poor survival (15-17). With the advent of global gene expression profiling, this tumor subgroup, termed basal-like, has garnered renewed interest in recent years due to the fact that these tumors display a distinct transcriptional profile (18) that correlates with a poor prognosis $(6,19,20)$. In addition, a link between this tumor subgroup and genotype has shown that hereditary breast cancer tumors with mutations in breast cancer 1, early onset (BRCA1) almost invariably display the basal-like phenotype (19). Although the basal-like subgroup appeared distinct when defined using gene expression data in a number of studies $(6,18-20)$, it should be noted that the formal definition and molecular markers that specify a basal-like tumor are still evolving (21-23).

Moyano and colleagues (9) use an immunohistochemistry-based (IHCbased) panel of protein expression (22) to identify the basal-like subgroup (estrogen receptor-negative [ER-negative], ErbB2/ HER-2-negative, and CK5/6-positive and/ or EGFR-positive). In this context, the association between $\alpha \mathrm{B}$-crystallin and the basal-like subtype of breast cancer, while attractive, is not as conclusive as the rest of their study. About half of the basal-like tumors characterized in a study by Sorlie 
et al. (6) and in the Moyano et al. study (9) did not overexpress $\alpha \mathrm{B}$-crystallin (mRNA and protein, respectively). Furthermore, about half of the $\alpha B$-crystallin IHC-positive tumors identified by Moyano et al. did not belong to their identified basal-like group. It is unknown whether the nonbasal-like, $\alpha \mathrm{B}$-crystallin-positive tumors are misclassified basal-like cases, or whether they are found randomly among the other breast cancer subtypes or enriched in certain subtypes. It should be noted that approximately half of the tumors belonging to the so-called "normal-like" subtype (which does not have such a poor prognosis as the basal-like subtype) have also been shown to express $\alpha \mathrm{B}$-crystallin message (6). That being said, the imperfect correlation between basal-like breast tumor marker expression and $\alpha \mathrm{B}$-crystallin expression may actually provide support for the idea that $\alpha \mathrm{B}$-crystallin is an oncogene, as it would be unlikely for an oncogene to be activated in all tumors of the imperfectly homogeneous basal-like subgroup. Nevertheless, Moyano et al. state that the $\alpha \mathrm{B}$-crystallin-transformed tumors that grew in the nude mice displayed basal-like characteristics; however, just how well these MCF-10A cell-derived tumors recapitulate the basal-like phenotype is not clear, as specific staining for basal CKs was not performed, nor were they analyzed for basal-like gene expression patterns. In this respect, any result should be interpreted within the context that MCF-10A cells themselves already appear to display a basal-like gene expression profile $(24,25)$. Additionally, while the authors show that $\alpha \mathrm{B}$-crystallin protein expression was associated with a shorter disease-specific survival independent of tumor grade, lymph node status, and ER and ErbB2 expression, it is not clear whether the prognostic value of $\alpha \mathrm{B}$-crystallin is independent of basal-like status. It also remains to be validated whether $\alpha \mathrm{B}$ crystallin is a general prognostic marker (in absence of therapy), a predictive marker for response to a particular therapy, both, or neither, as the patients studied by Moyano et al. (9) were diagnosed over several decades and had substantially varied (and unspecified) adjuvant therapies (26). This is particularly important given that increased expression of $\alpha \mathrm{B}$-crystallin in cell line models has been associated with acquired resistance to the DNA-damaging agents cisplatin, etoposide, and fotemustine (27) and in light of recent evidence that the basal-like, but not the normallike, subtype is particularly sensitive to preoperative paclitaxel followed by 5 -fluorouracil, doxorubicin, and cyclophosphamide chemotherapy (28).

As with any groundbreaking work, the study by Moyano and colleagues (9) opens up as many questions as it convincingly answers. Their impressive study marks a significant step forward in our understanding of how $\alpha \mathrm{B}$-crystallin contributes to cancer; clearly, $\alpha \mathrm{B}$-crystallin is involved in many more ways than simply suppressing apoptosis. Furthermore, if validation studies demonstrate that human breast tumors with high $\alpha \mathrm{B}$-crystallin levels have an activated ERK/MAPK pathway, then a tailored therapy for this phenotype may soon be clinically testable, as at least 2 drugs active against this pathway are currently being evaluated: CI-1040 (also known as PD 184352), an orally available and well-tolerated MEK1/2 inhibitor, has shown encouraging results in phase I and II clinical trials as an anticancer agent (29), and PD 0325901, a second-generation MEK inhibitor, with which even better responses are anticipated following completion of a National Cancer Institute Clinical Trial (NCT00147550).

Address correspondence to: Ramon Parsons, Departments of Pathology and Medicine, Institute for Cancer Genetics, Herbert Irving Comprehensive Cancer Center, Columbia University College of Physicians and Surgeons, 1150 St. Nicholas Avenue, Russ Berrie Pavilion, New York, New York 10032, USA. Phone: (212) 851-5278; Fax: (212) 851-5256; E-mail: rep15@columbia.edu.

1. Clark, J.I., and Muchowski, P.J. 2000. Small heatshock proteins and their potential role in human disease. Curr. Opin. Struct. Biol. 10:52-59.

2. Parcellier, A., et al. 2005. Small heat shock proteins HSP27 and alphaB-crystallin: cytoprotective and oncogenic functions. Antioxid. Redox Signal. 7:404-413.

3. Sun, Y., and MacRae, T.H. 2005. The small heat shock proteins and their role in human disease. FEBS J. 272:2613-2627.

4. Aoyama, A., et al. 1993. Expression of alpha Bcrystallin in human brain tumors. Int. J. Cancer. 55:760-764.

5. Pinder, S.E., et al. 1994. The expression of alpha B-crystallin in epithelial tumours: a useful tumour marker? J. Pathol. 174:209-215.

6. Sorlie, T., et al. 2001. Gene expression patterns of breast carcinomas distinguish tumor subclasses with clinical implications. Proc. Natl. Acad. Sci.U. S. A. 98:10869-10874.

7. Chelouche-Lev, D., Kluger, H.M., Berger, A.J., Rimm, D.L., and Price, J.E. 2004. alphaB-crystallin as a marker of lymph node involvement in breast carcinoma. Cancer. 100:2543-2548.

8. Wulfkuhle, J.D., et al. 2002. Proteomics of human breast ductal carcinoma in situ. Cancer Res. 62:6740-6749.

9. Moyano, J.V., et al. 2006. $\alpha \mathrm{B}$-Crystallin is a novel oncoprotein that predicts poor clinical outcome in breast cancer. J. Clin. Invest. 116:261-270. doi:10.1172/JCI25888.

10. Zhou, Q., et al. 2000. Cyclin D1 overexpression in a model of human breast premalignancy: preferential stimulation of anchorage-independent but not anchorage-dependent growth is associated with increased cdk2 activity. Breast Cancer Res. Treat. 59:27-39.

11. Ciardiello, F., et al. 1992. Additive effects of c-erbB-2, c-Ha-ras, and transforming growth factor-alpha genes on in vitro transformation of human mammary epithelial cells. Mol. Carcinog. 6:43-52.

12. Miller, F.R., Pauley, R.J., and Wang, B. 1996. Activated c-Ha-ras is not sufficient to produce the preneoplastic phenotype of human breast cell line MCF10AT. Anticancer Res. 16:1765-1769.

13. Li, D.W., et al. 2005. Calcium-activated RAF/MEK/ ERK signaling pathway mediates p53-dependent apoptosis and is abrogated by alphaB-crystallin through inhibition of RAS activation. Mol. Biol. Cell. 16:4437-4453.

14. Chen, Z., et al. 2001. MAP kinases. Chem. Rev. 101:2449-2476.

15. Moll, R., Franke, W.W., Schiller, D.L., Geiger, B., and Krepler, R. 1982. The catalog of human cytokeratins: patterns of expression in normal epithelia, tumors and cultured cells. Cell. 31:11-24.

16. Moll, R., Krepler, R., and Franke, W.W. 1983. Complex cytokeratin polypeptide patterns observed in certain human carcinomas. Differentiation. 23:256-269.

17. Dairkee, S.H., Mayall, B.H., Smith, H.S., and Hackett, A.J. 1987. Monoclonal marker that predicts early recurrence of breast cancer [letter]. Lancet. 1:514.

18. Perou, C.M., et al. 2000. Molecular portraits of human breast tumours. Nature. 406:747-752.

19. Sorlie, T., et al. 2003. Repeated observation of breast tumor subtypes in independent gene expression data sets. Proc. Natl. Acad. Sci. U. S. A. 100:8418-8423.

20. Sotiriou, C., et al. 2003. Breast cancer classification and prognosis based on gene expression profiles from a population-based study. Proc. Natl. Acad. Sci. U. S. A. 100:10393-10398.

21. van de Rijn, M., et al. 2002. Expression of cytokeratins 17 and 5 identifies a group of breast carcinomas with poor clinical outcome. Am. J. Pathol. 161:1991-1996.

22. Nielsen, T.O., et al. 2004. Immunohistochemical and clinical characterization of the basal-like subtype of invasive breast carcinoma. Clin. Cancer Res. 10:5367-5374.

23. Arnes, J.B., et al. 2005. Placental cadherin and the basal epithelial phenotype of BRCA1-related breast cancer. Clin. Cancer Res. 11:4003-4011.

24. Ross, D.T., and Perou, C.M. 2001. A comparison of gene expression signatures from breast tumors and breast tissue derived cell lines. Dis. Markers. 17:99-109.

25. Charafe-Jauffret, E., et al. 2006. Gene expression profiling of breast cell lines identifies potential new basal markers. Oncogene. In press.

26. Makretsov, N.A., et al. 2004. Hierarchical clustering analysis of tissue microarray immunostaining data identifies prognostically significant groups of breast carcinoma. Clin. Cancer Res. 10:6143-6151.

27. Wittig, R., et al. 2002. Candidate genes for crossresistance against DNA-damaging drugs. Cancer Res. 62:6698-6705.

28. Rouzier, R., et al. 2005. Breast cancer molecular subtypes respond differently to preoperative chemotherapy. Clin. Cancer Res. 11:5678-5685.

29. Rinehart, J., et al. 2004. Multicenter phase II study of the oral MEK inhibitor, CI-1040, in patients with advanced non-small-cell lung, breast, colon, and pancreatic cancer. J. Clin. Oncol. 22:4456-4462. 